2012-5

\title{
Modelling Two-Dimensional Photopolymer Patterns Produced With Multiple-Beam Holography
}

\author{
Dana Mackey \\ Technological University Dublin, dana.mackey@tudublin.ie \\ Tsvetanka Babeva \\ Bulgarian Academy of Sciences, babeva@iomt.bas.bg \\ Izabela Naydenova \\ Technological University Dublin, izabela.naydenova@tudublin.ie
}

See next page for additional authors

Follow this and additional works at: https://arrow.tudublin.ie/scschmatcon

Part of the Optics Commons, and the Partial Differential Equations Commons

\section{Recommended Citation \\ 978-3-642-25100-9_43 \\ This Conference Paper is brought to you for free and open access by the School of Mathematics at ARROW@TU Dublin. It has been accepted for inclusion in Conference papers by an authorized administrator of ARROW@TU Dublin. For more information, please contact arrow.admin@tudublin.ie, aisling.coyne@tudublin.ie,gerard.connolly@tudublin.ie.}

Mackey, D., Babeva, T., \& Naydenova, I. (2012). Modelling Two-Dimensional Photopolymer Patterns Produced With Multiple-Beam Holography. 16th European Conference on Mathematics for Industry, July 26th-30th, 2010 Wuppertal, Germany. Mathematics in Industry, vol. 17. doi:10.1007/

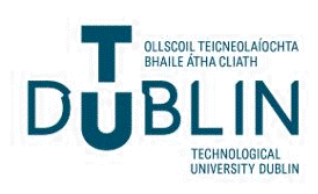




\section{Authors}

Dana Mackey, Tsvetanka Babeva, Izabela Naydenova, and Vincent Toal 


\title{
Modelling two-dimensional photopolymer patterns produced with multiple-beam holography
}

Dana Mackey, Tsvetanka Babeva, Izabela Naydenova and Vincent Toal

\begin{abstract}
Periodic structures referred to as photonic crystals attract considerable interest due to their potential applications in areas such as nanotechnology, photonics, plasmonics, etc. Among various techniques used for their fabrication, multiplebeam holography is a promising method enabling defect-free structures to be produced in a single step over large areas.

In this paper we use a mathematical model describing photopolymerisation to simulate two-dimensional structures produced by the interference pattern of three noncoplanar beams. The holographic recording of different lattices is studied by variation of certain parameters such as beam wave vectors, time and intensity of illumination.
\end{abstract}

\section{Introduction}

Grating evolution in photopolymers has been studied by several authors ( [1], [2], [3], etc.). It is known that exposing a photopolymer material to an illumination pattern causes light-induced mass transport of the system components. The recorded

Dana Mackey

School of Mathematical Sciences, Dublin Institute of Technology, Kevin Street, Dublin 8, Ireland, e-mail: dana.mackey@dit.ie

Tsvetanka Babeva

Institute of Optical Materials and Technologies, Bulgarian Academy of Sciences, Sofia, Bulgaria, e-mail: babeva@clf.bas.bg

Izabela Naydenova

Centre for Industrial and Engineering Optics, Dublin Institute of Technology, Ireland,

e-mail: izabela.naydenova@dit.ie

Vincent Toal

Centre for Industrial and Engineering Optics, Dublin Institute of Technology, Ireland, e-mail: vincent.toal@dit.ie 
holographic grating is then due to a spatial variation of the refractive index resulting from changes in the density of the monomer and polymer species. However, the common feature of most theoretical models proposed to date is that they fail to describe the experimental observation of poor diffraction efficiency at high spatial frequencies. A two-way diffusion theory was proposed in [4] and [5] and states that the counter diffusion of short-chain polymer molecules away from the bright fringes is responsible for the reduction in diffraction efficiency. To verify this assumption, a new mathematical model for the formation of a weak grating after short exposure times was proposed in [6], [7]. This model accounts for both monomer and polymer diffusion and moreover distinguishes between short polymer chains capable of diffusing and long polymer chains that are immobile. The time evolution of refractive index modulation was calculated and compared with experimental results for different values of the diffusion coefficients, polymerization rates, intensity and spatial frequency of recording. It was concluded that the two-way diffusion model successfully predicts the observed grating deterioration at high frequencies and this has been further validated by good agreement within other experimental settings, [8].

In this paper, the model is generalised to describe two-dimensional holographic gratings and preliminary results regarding numerical investigations of photopolymerisation patterns produced by the interference pattern of three noncoplanar beams are presented.

\section{Three-beam holography}

Procedures for implementing 2-dimensional optical interference profiles through holography, together with strategies for optimising the desired lattice characteristics in terms of propagation and polarisation parameters can be found in the literature (see, for example, [9], [10], [11]).

In this work we restrict our study to patterns produced by three non-coplanar beams with electric field vectors

$$
\mathbf{E}_{m}(\mathbf{r})=E_{m} \exp \left(i \mathbf{K}_{m} \cdot \mathbf{r}+i \delta_{m}\right) \mathbf{e}_{m}, m=1,2,3,
$$

where $E_{m}$ is the real amplitude, $\delta_{m}$ is the absolute phase and $\mathbf{e}_{\mathbf{m}}$ are unit vectors which define the polarisation directions. The propagation vectors

$$
\mathbf{K}_{m}=\frac{2 \pi}{\lambda}\left(\cos \left(\phi_{m}\right) \sin \left(\theta_{m}\right), \sin \left(\phi_{m}\right) \sin \left(\theta_{m}\right), \cos \left(\theta_{m}\right)\right)
$$

are expressed in the spherical coordinate system illustrated in Fig. 1. ( $\lambda$ is the recording wavelength.) The illumination intensity is defined as the square of the total electric field and, for the case of linearly polarised waves, given in [10] as

$$
\mathbf{I}(\mathbf{r})=\sum_{l=1}^{3} E_{l}^{2}+\sum_{1=l<m}^{3} 2 E_{l} E_{m} \cos \theta_{l m} \cos \left(\left(\mathbf{K}_{l}-\mathbf{K}_{m}\right) \cdot \mathbf{r}+\delta_{l}-\delta_{m}\right)
$$




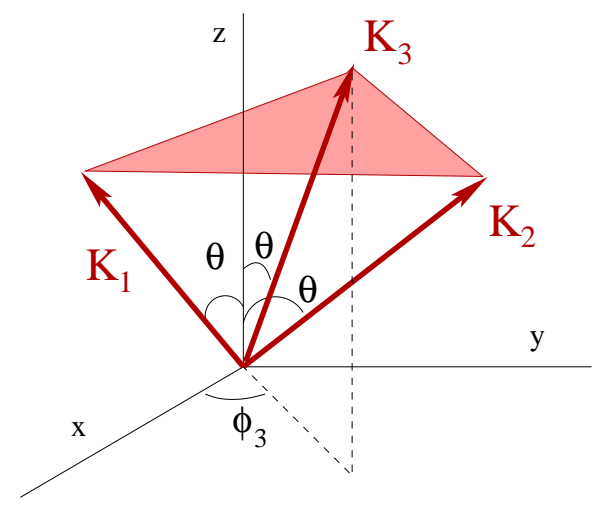

Fig. 1 The geometry of the three beams

where $\theta_{l m}$ is the angle between $\mathbf{K}_{l}$ and $\mathbf{K}_{m}$. Since the interference pattern resulting from three noncollinear propagation vectors is always two-dimensional, we choose the coordinate system so that all polar propagation angles are equal, $\theta_{1}=\theta_{2}=\theta_{3} \equiv$ $\theta$, and one reciprocal wave vector, say $K_{2}-K_{1}$, is parallel to the $y$-axis. The intensity can then be conveniently expressed as

$$
I(x, y)=I_{0}\left[1+\sum_{1=l<m}^{3} V_{l m} \cos \left(A_{l m} x+B_{l m} y\right)\right]
$$

where

$$
\begin{gathered}
A_{l m}=\frac{2 \pi}{\lambda}\left(\cos \phi_{l}-\cos \phi_{m}\right) \sin \theta, \quad B_{l m}=\frac{2 \pi}{\lambda}\left(\sin \phi_{l}-\sin \phi_{m}\right) \sin \theta \\
\frac{3}{2} V_{l m}=\cos \left(\phi_{l}-\phi_{m}\right)\left[\cos ^{2} \theta \cos \psi_{l} \cos \psi_{m}+\sin \psi_{l} \sin \psi_{m}\right] \\
-\cos \theta \sin \left(\phi_{l}-\phi_{m}\right) \sin \left(\psi_{l}-\psi_{m}\right)+\sin ^{2} \theta \cos \psi_{l} \cos \psi_{m}
\end{gathered}
$$

The maximum intensity points form a two-dimensional lattice with primitive vectors

$$
\mathbf{a}_{1}=\frac{\lambda}{\sin (\theta)\left(\cos \left(\phi_{1}\right)-\cos \left(\phi_{2}\right)\right)}(1,0), \quad \mathbf{a}_{2}=\frac{\lambda}{2 \sin (\theta) \sin \left(\phi_{2}\right)}\left(\cot \left(\frac{\phi_{1}+\phi_{2}}{2}\right), 1\right)
$$

and we let $a=\left|\mathbf{a}_{1}\right|$ and $b=\left|\mathbf{a}_{2}\right|$.

\section{Two-dimensional diffusion-polymerisation model}

The model proposed in [7], [6] takes into account monomer and polymer diffusion, creation of short polymer chains and introduces a simple "immobilization" mecha- 
nism which mimics the growth of polymer chains to the extent where they cannot diffuse any longer. This diffusion-polymerisation model consists of partial differential equations for three unknown functions which represent the concentrations of monomer, $m$, short polymer chains, $p_{1}$ and long polymer chains, $p_{2}$, which is easily generalised to two dimensions.

$$
\begin{aligned}
& \frac{\partial m}{\partial t}=D_{m} \Delta m-\Phi(t) F(x, y) m \\
& \frac{\partial p_{1}}{\partial t}=\nabla\left(D(x, y) \nabla p_{1}\right)+\Phi(t)\left[F(x, y) m-\gamma m p_{1}\right] \\
& \frac{\partial p_{2}}{\partial t}=\Phi(t) \gamma m p_{1},
\end{aligned}
$$

The spatial domain is chosen as $-a \leq x \leq a,-b \leq y \leq b$ and $t \geq 0$. Here $D_{m}$ is the monomer diffusion constant and, as justified in [6], we assume that the short polymer diffusion coefficient, as well as the polymerization rate are proportional to the illumination, $D(x, y)=D_{p} I(x, y)$ and $F(x, y)=F_{0} I(x, y)$, where $I(x, y)$ is given by (2) and $F_{0}$ is the polymerization constant.

We assume the initial conditions are given by $m(x, 0)=m_{0}$ and $p_{1}(x, 0)=$ $p_{2}(x, 0)=0$. To account for a finite exposure time, $t_{e}$, we have introduced the step function $\Phi(t)=1$ if $t \leq t_{e}$ and $\Phi(t)=0$ if $t>t_{e}$. For convenience with the numerical simulations, the system is then non-dimensionalised by choosing

$$
\bar{x}=\frac{x}{a}, \quad \bar{y}=\frac{y}{b}, \quad \bar{m}=\frac{m}{m_{0}}, \quad \bar{p}_{i}=\frac{p_{i}}{m_{0}}(i=1,2) .
$$

The refractive index of a material consisting of a mixture of components can be calculated with the well-known Lorentz-Lorenz equation,

$$
\frac{n^{2}-1}{n^{2}+2}=\sum_{i} \Phi_{i} \frac{n_{i}^{2}-1}{n_{i}^{2}+2}
$$

where $n$ is the effective refractive index of the mixture, $n_{i}$ are the refractive indices of the components (monomer, polymer and binder) determined experimentally, and $\Phi_{i}$ are the normalized concentrations of the components. (For more details of this calculation see, for example, [6].)

\section{Numerical results and conclusions}

The non-dimensional model was integrated numerically using a finite difference ADI (Alternating Direction Implicit) scheme. We choose a simple interference pattern based on three linearly polarized beams with the propagation and polarization parameters chosen as in [9]: $\theta=0.384, \phi_{1}=\frac{\pi}{4}, \phi_{2}=\frac{3 \pi}{4}, \phi_{3}=-\frac{3 \pi}{4}, \psi_{1}=2.56$, $\psi_{2}=0.178, \psi_{3}=0.918$. This beam configuration gives rise to an illumination pat- 
tern characterized by a square lattice (since the primitive vectors $\mathbf{a}_{1}, \mathbf{a}_{2}$ are orthogonal and $a=b$ ) and, in this context, the partial differential equations model presented in the previous section can be supplemented by zero-flux boundary conditions. The recording wavelength was kept constant at $\lambda=532 \times 10^{-9} \mathrm{~m}$. The numerical values used for the diffusion constants are $D_{m}=10^{-7} \mathrm{~cm}^{2} / \mathrm{s}, D_{p}=10^{-9} \mathrm{~cm}^{2} / \mathrm{s}$ and the polymerization rate constant was varied between $F_{0}=0.01-10 \mathrm{~s}^{-1}$. The exposure time is $t_{e}=5 \mathrm{~s}$ and the system was integrated for a total of 50s.

The spatial distribution of the refractive index was calculated from (3), as a function of the monomer and polymer concentrations, at various times during and after exposure and the resulting grating formation was compared with the illumination pattern. It was found that, in general, the accuracy of copying was good except for parameter regimes where diffusion times were much smaller than polymerisation times. (This effect could be illustrated, for example, by decreasing the polymerisation rate $F_{0}$ - see Figure 2). Figure (3) shows spatial concentration profiles of long polymers obtained after exposure for two values of $F_{0}$. It is noted that for low values of $F_{0}$ (or other parameter combinations which result in low ratios of diffusion to polymerisation times), the concentration profiles of long polymers develop contributions from higher harmonics. (This is in accordance with a similar observation made in [7] for the case of the one-dimensional illumination pattern.)

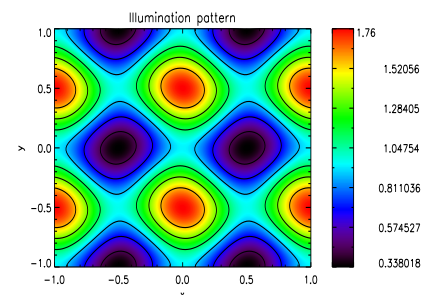

(a) Illumination pattern

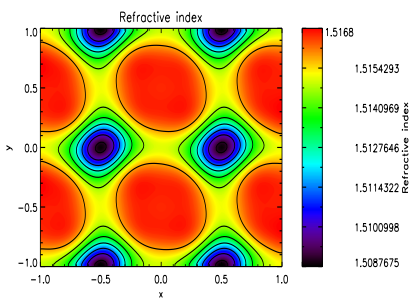

(c) $F_{0}=0.1$

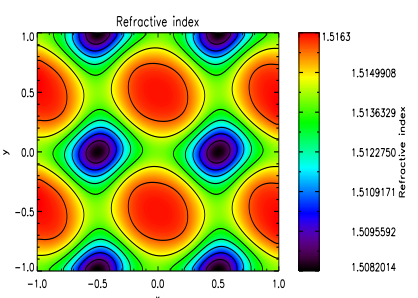

(b) $F_{0}=1$

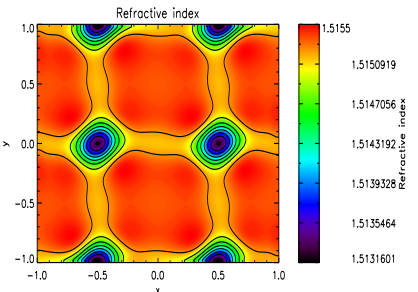

(d) $F_{0}=0.01$

Fig. 2 Comparison of illumination pattern and refractive index patterns after exposure

In conclusion, a preliminary theoretical and computational study was presented with the purpose of assessing the suitability of a mathematical model for describing gratings produced with multiple beam holography. More such simulations are needed in order to assess the influence of various parameters (related to both the 


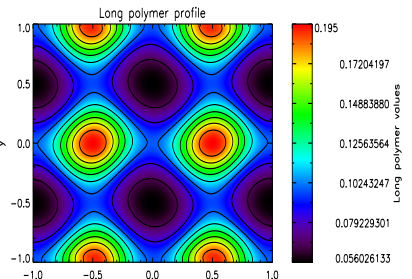

(a) $F_{0}=0.1$

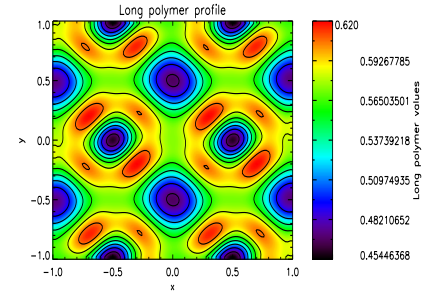

(b) $F_{0}=0.01$

Fig. 3 Spatial distribution of long polymers after exposure

photopolymer system and the recording process) on the accuracy of copying a twodimensional light pattern into a change of the refractive index and such research is currently being carried out by our team.

\section{References}

1. Piazzola, S.and Jenkins, B. : First-harmonic diffusion model for holographic grating formation in photopolymers, J. Opt. Soc. Am. B 17, 1147-1157 (2000)

2. Zhao, G. and Mouroulis, P.: Diffusion model of hologram formation in dry photopolymer materials, J. Mod. Opt. 41, 1929-1939 (1994)

3. Colvin, V.L., Larson, R.G., Harris, A.L. and Schilling, M.L.: Quantitative model of volume hologram formation in photopolymers, J. Appl. Phys. 81, 5913-5923 (1997)

4. Martin, S., Naydenova, I., Jallapuram, R., Howard, R. and Toal, V.: Two-way diffusion model for the recording mechanism in a self developing dry acrylamide photopolymer, Proc. SPIE 6252, 62525-625217 (2006)

5. I. Naydenova, R. Jallapuram, R. Howard, S. Martin, and V. Toal, Investigation of the Diffusion Processes in a Self-Processing Acrylamide-Based Photopolymer System, Appl. Opt. 43, 29002905 (2004).

6. Babeva, T., Naydenova, I., Mackey, D., Martin, S. and Toal, V.: Two-way diffusion model for short exposure holographic grating, formation in acrylamide based photopolymers, Journal of the Optical Society of America B, Vol 27, No. 2, (2010)

7. Mackey, D., Babeva, T., Naydenova, I. and Toal, V., Babeva, T., Naydenova, I. and Toal, V.: A diffusion model for spatially dependent photopolymerisation. In: A.D. Fitt, J. Norbury, H. Ockendon, E. Wilson (eds.) Progress in Industrial Mathematics at ECMI 2008, Mathematics in Industry, vol. 15, pp. 253-259. Springer, Berlin Heidelberg New York (2010)

8. T. Babeva, D.Mackey, I.Naydenova, S.Martin, V.Toal, Study of the photoinduced surface relief modulation in photopolymers caused by illumination with a Gaussian beam of light, Journal of Optics, Vol. 12, 2010;

9. Escuti, M.J., Crawford, G.P. : Holographic photonic crystals, Optical Engineering, 43 (9), pp.1973-1987 (2004)

10. Cai, L.Z., Yang, X.L. and Wang, Y.R.: Formation of a microfiber bundle by interference of three noncoplanar beams, Optics Letters, 26 (23), pp. 1858-1860, (2001)

11. Mao, W., Zhong, Y., Dong, J. and Wang, H.: Crystallography of two-dimensional photonic lattices formed by holography of three noncoplanar beams, Journal of the Optical Society of America B, 22 (5), pp. 1085-1091, (2005) 